Article

\title{
An Evaluation of Symmetries in Ground Reaction Forces during Self-Paced Single- and Dual-Task Treadmill Walking in the Able-Bodied Men
}

\author{
Rawan Al-Juaid ${ }^{1}$ and Mohammad Al-Amri ${ }^{1,2, *(D)}$ \\ 1 School of Healthcare Sciences, College of Biomedical and Life Sciences, Cardiff University, \\ Cardiff CF24 0AB, UK; AlJuaidRM@cardiff.ac.uk \\ 2 Biomechanics and Bioengineering Research Centre Versus Arthritis, Cardiff University, \\ Cardiff CF10 3AX, UK \\ * Correspondence: al-amrim@cardiff.ac.uk
}

Received: 30 November 2020; Accepted: 16 December 2020; Published: 17 December 2020

\begin{abstract}
Gait is a complex autonomous activity that has long been viewed as a symmetrical locomotion, even when it adapts to secondary concurrent attention-demanding tasks. This study aimed to evaluate the symmetry of the three ground reaction forces (GRFs) in able-bodied individuals during self-paced treadmill walking with and without concurrent cognitive demands. Twenty-five male participants (age: $34.00 \pm 4.44$ years) completed two gait assessment sessions, each of whom were familiarized with the walking trials during their first session. Both sessions involved six-minute self-paced treadmill walking under three conditions: single-task walking and walking while concurrently responding to auditory 1-back and 2-back memory tasks. The symmetry of the GRFs was estimated using a nonlinear approach. Changes in the symmetry and walking speed across conditions in both sessions were assessed using inferential statistics. Results demonstrated that the three GRFs deviated from perfect symmetry by $\geq 10 \%$. Engaging working memory during walking significantly reduced the symmetry of the vertical GRF $(p=0.003)$, and its detrimental effects on walking speed were significantly reduced in the second session with respect to the first session $(p<0.05)$. The findings indicate imperfect gait symmetry in able-bodied individuals, suggesting that common perceptions of gait symmetry should be reconsidered to reflect its objective importance in clinical settings.
\end{abstract}

Keywords: gait symmetry; ground reaction forces; dual-task walking; healthy individuals; treadmill-based gait analysis

\section{Introduction}

Gait symmetry describes the agreement between the movement of the right and left lower extremities in pattern and proportion [1]. Symmetry is a critical aspect of gait because it makes gait a seemingly smooth, functional, and dynamically stable process with minimal mechanical demands [1-4]. Due to the symmetrical appearance of able-bodied individuals' gait, symmetry has been taken for granted for a long time to represent typical healthy gait patterns, while asymmetry in gait has been considered undesirable. Gait symmetry assessment is often used in clinical practice to plan and measure the effectiveness of rehabilitation programs. Despite its importance, it is routinely assessed by clinicians using observational techniques, including naked eye assessment and video recordings [5]. Visual gait assessments can detect obvious deviations from normal patterns but not necessarily determine the primary deficits $[5,6]$. Therefore, these types of assessment are neither sensitive nor reliable enough to assess gait symmetry, especially for people with symmetrical-looking gait performance. 
Over the past few decades, various gait parameters, including kinetics [7-9], kinematics [10,11], electromyography profiles [12], and spatiotemporal variables [10], have been examined to investigate whether able-bodied individuals' gait is actually symmetrical or not. However, gait symmetry in able-bodied individuals is still a matter of debate $[1,13]$. The contradictory perspectives might be attributed to the absence of standardization of how gait symmetry in able-bodied individuals should be assessed and which gait parameters are more appropriate to examine [1,4,13]. Of all gait parameters, the ground reaction forces (GRFs) have been the least examined, although they are reliable, relatively easy to obtain in clinical settings, and more informative than other parameters $[14,15]$. The examination of the GRF parameters during walking can provide information about the reaction forces acting on the human body in three directions: vertical, anterior-posterior, and medial-lateral [15]. Each of these directions is of clinical importance. The vertical GRF supports the body against the gravitational force to prevent the body from collapsing, while the anterior-posterior GRF affects the acceleration and deceleration of locomotion and the medial-lateral GRF affects the dynamic balance [15]. Previous studies only examined selected discrete parameters of GRF in able-bodied participants during nonconsecutive or minimal consecutive overground walking strides $[7-9,12,16,17]$. The examination of selected parameters does not provide an overall insight into symmetry in able-bodied individuals' gait due to the risk of neglecting some hidden asymmetrical features within the gait cycles [13]. Currently, more continuous GRF waveforms can be obtained during natural consecutive gait cycles owing to the recent advancements in self-paced instrumented treadmills, which can be advantageous to overcome methodological limitations in existing literature [7-9,12,16,17]. These cutting-edge treadmills offer comprehensive gait analysis [18], and their reliability to collect valid gait parameters that are comparable to overground walking has been examined [19]. Despite the growing use of these treadmills in biomechanical research, no published work has evaluated the symmetry characteristics of the GRF parameters in able-bodied individuals during self-paced treadmill walking. Treadmill-based gait analysis offers normative gait parameters that would provide better insights into the mechanisms underlying gait coordination.

Gait was believed to be an automated process that requires minimal cognitive demands [20]. Nevertheless, a growing body of evidence suggests that more attentional resources are needed during walking while concurrently performing another motor or cognitive task-so-called dual-task walking. Dual-task walking is a commonly used paradigm to investigate the cognitive contributions to gait control by evaluating the attentional costs on gait parameters after introducing a concurrent cognitive task to walking, relative to the baseline walking condition [20]. Although dual-task walking is an essential skill for safe and independent ambulation during daily functional activities [21,22], evidence shows that dual-task walking impacts on some gait aspects even among healthy young adults, who were found to walk more slowly and with a greater variability during dual-tasking [23,24]. Schaefer and Lindenberger [25] reported that women who habitually wear high heels adapt their gait with greater flexibility and less variability than do novice women during dual-task walking. These findings imply that gait adaptation during unfamiliar walking conditions might not be as easily and efficiently performed as that under well-familiarized conditions, even amongst people of the same age and characteristics. Accordingly, the lack of familiarization prior to the examination of dual-task effects could cause some confusion regarding whether the significance of dual-task effects on gait was originally caused by dividing attention between two concurrent tasks or was just a matter of unfamiliarity with the nature of the walking condition. Therefore, to avoid misleading results, it is important to provide participants with sufficient time to familiarize themselves with the nature of walking conditions.

Despite the broad research in gait symmetry, only a few studies have examined the effects of dual-task walking on gait symmetry [26-28]. In these studies, spatiotemporal parameters (e.g., swing time) were quantified in patients with Parkinson's disease (PD) [28], idiopathic elderly fallers, and healthy elderly subjects $[26,27]$ to assess gait asymmetry under single- and dual-task walking. Yogev et al. [26] reported a significant increase in the asymmetric values during dual-task walking amongst the symptomatic subjects. In contrast, Ribeiro et al. [28] did not observe a significant effect on 
the gait asymmetry of PD subjects under a dual-task walking condition. A definitive conclusion as to whether cognitive loading during dual-task walking could impact gait symmetry cannot be extracted. Therefore, further research is imperative, especially in the able-bodied population, to eliminate confounding factors associated with the disease type or its stage. To date, no study has examined the effects of dual-task walking on the symmetry of the GRFs in able-bodied individuals' gait. This is fundamental information, which is needed to provide clinicians with reference values to differentiate between normal and pathological asymmetries in gait. Hence, the objectives of this study were (1) to establish the symmetry characteristics of the vertical, anterior-posterior, and medial-lateral GRFs parameters in able-bodied individuals over two self-paced treadmill gait assessment sessions while walking under three conditions (a single-task walking and two dual-task walking conditions), (2) to examine between-session differences in symmetry and speed parameters across walking conditions, and (3) to investigate whether concurrent memory tasks would significantly impact the symmetry and speed of able-bodied individuals' gait.

\section{Materials and Methods}

\subsection{Participants}

The gait dataset used in this study was extracted from a larger study exploring self-paced treadmill walking in able-bodied participants [18]. Briefly, participants were males aged between 30 and 50 years old to yield a well-defined able-bodied population with similar physical capabilities, which were necessary to eliminate confounding factors influencing symmetry, such as age, gender, or a certain pathology [1]. Participants were included in the study only if they presented with no previous history of any medical, neurological, musculoskeletal, cardiovascular, or vestibular pathologies that might affect gait health. Participants were excluded if they reported any cognitive impairments, learning difficulties, or impaired normal vision. Ethical approval for the study was granted by the Cardiff University School of Healthcare Sciences Research Ethics Committee. Participation was entirely voluntary, and written informed consent was acquired prior to participation. A powered sample size of a minimum of 17 participants was determined through $G^{*}$ Power software (version 3.1.9.3) [29]. Power was set at 0.8 , with a medium effect size $(f=0.30)$ and an $\alpha$ level of 0.05 . However, we chose to evaluate the data from 25 participants, given that this is twice the number of male participants in many previous studies $[8,9,17]$.

\subsection{Experimental Procedure}

All participants underwent two separate self-paced treadmill gait analysis sessions, separated by $5 \pm 3$ days. During each session, the participants completed three walking trials: free single-task walking (FW), and walking while concurrently performing the 1-back task (DT1) and the 2-back task (DT2). Bilateral GRF parameters and average walking speed were recorded via the Gait Real-time Analysis Interactive Lab (GRAIL; Motekforce Link, Amsterdam, The Netherlands) system at Cardiff University [18]. The GRAIL system consists of a reliable split-belt instrumented treadmill with two embedded force plates which were used to obtain bilateral GRFs at a sampling rate of $2000 \mathrm{~Hz}$ [18]. This instrumented treadmill is capable of adapting to the self-paced walking speed of each participant [30], whilst a synchronized virtual walking scene was presented on a semicylindrical screen and surrounded by 12 optical infrared cameras to capture movement (Vicon, Oxford Metrics, Oxford, UK) [31]. Both belts were set to equal speeds, and their speeds were based on the self-paced walking state for each participant to allow them to intrinsically control the treadmill's speed [18]. The self-paced algorithm was described by Sloot et al. [32]. The speed of the visual flow on the virtual reality screen was maintained by the walking speed [18].

All participants were requested to wear shorts and aqua shoes for their safety and convenience, and each of them was prepared individually for the walking trials [18]. Twenty-five reflective markers were placed over the bony landmarks of the participant's lower limbs according to the human body 
model (HBM) [31]. Each participant also wore a safety harness that dangled from the ceiling to ensure safety during their treadmill walking. The position of the harness allowed the participant to walk without restricting their normal walking state. Subsequently, the participant was asked to stand in the middle of the treadmill, and each foot was positioned on a corresponding force plate. The participant was then instructed to walk at their comfortable speed on the treadmill for a minimum of $6 \mathrm{~min}$ to ensure enough time for familiarization, as suggested by Meyer et al. [33]. The participants then completed three walking trials, each of which lasted $6 \mathrm{~min}$. The order of the walking trials was randomized to avoid any learning effect [34]. A 2 min rest period was provided between walking trials to avoid the risk of fatigue [34]. The same protocol was repeated for the same participant in session 2 after practicing self-paced walking on the treadmill for 3 mins to avoid getting rusty. For this study, we considered the assessment during the first session as part of the familiarization with the tested protocol to ensure that participants were well rested prior the actual assessment of the dual-task effects on gait symmetry. However, we captured the data during both sessions to assess whether there were significant differences between the measurements recorded during the familiarization session and the testing session.

During dual-task waking, the auditory $N$-back memory task was introduced as the secondary task for two main reasons; firstly, the concurrent performance of the $\mathrm{N}$-back task while walking was found to induce significant effects on the speed, stride length, and cadence of a comparable healthy adult population [35]. These effects indicate that the $N$-back would be a sufficiently attentional demanding task for our participants, which is necessary for the exploration of whether symmetry regulation in the able-bodied would have to compete with the $N$-back task for the attentional resources. Secondly, the complexity of walking conditions can be easily manipulated by increasing the size of $N$ (i.e., 0 , 1,2 , or 3 back) [36]. In this study, the 1-back and 2-back tests were used to manipulate the cognitive loading during dual-task walking conditions. Theoretically, the 2-back test poses a higher cognitive loading than the 1-back test [37]. In this study, the participants were provided with a handheld button, which they were instructed to press when the incoming auditory stimulus was an $N$-back repeat. The reliability of this methodology was previously demonstrated [36]. No specific instructions were given to the participants with regard to which task, the cognitive or walking task, should be prioritized during both dual-task walking conditions.

\subsection{Data Processing}

The data for the three GRFs and walking speed of the 25 participants during the three walking conditions in both sessions were processed using MATLAB (Version 2018b, MathWorks, Natick, MA, USA). A total of 100 strides located within a $4 \mathrm{~min}$ period (from 50-310 s of the entire walking duration-360 s) were evaluated. The strides at the beginning and the end of each walking trial were eliminated to negate the effects of gait initiation and termination [18]. The average walking speed for each walking condition was measured during this period.

Multiresolution entropy was utilized to quantify symmetry within the complete gait cycles during the three walking conditions in both sessions. Multiresolution entropy is a nonlinear symmetry approach that has advantages to overcome the limitations of relying only on discrete parameters to quantify symmetry $[13,38]$. The symmetry values range between 0 and 1 ; a value of " 1 " indicates perfect symmetry and a value of " 0 " indicates asymmetry [13]. For the interpretation of symmetry, Robinson et al. [39] and Liikavainio et al. [40] suggested that deviations by more than $10 \%$ from the perfect symmetry value could be classified as asymmetric [12]. Similarly, we decided to set the cut-off point of symmetry at 0.90 , with lower values indicating gait asymmetry.

\subsection{Statistical Analysis}

The symmetry and speed data were analyzed using descriptive and inferential statistical methods. The analysis aimed to investigate the between-session differences and locate the significance of any effect of concurrent $N$-back memory tasks on symmetry and walking speed. Descriptive information, including mean and standard deviation (SD), was reported for each symmetry score and walking 
speed across walking conditions. To promote better understanding of the data behavior, the coefficient of variation $(\mathrm{CV})$ for each GRF component and walking speed across walking conditions was also presented. The CV was calculated using the following formula:

$$
\mathrm{CV}=\frac{\mathrm{SD}}{\text { Mean }} \times 100
$$

After assessing the normality of the data distribution using the Kolmogorov-Smirnov test, Mauchly's test was used to check whether the sphericity assumptions of the repeated-measures analysis of variance (ANOVA) were satisfied [34]. Repeated-measures ANOVA was then used to investigate the significant change in variables among FW, DT1, and DT2. Repeated-measures ANOVA is a powerful tool that allows comparison of repeated measurements for each participant, and it was deemed a suitable model in a similar study [30]. Paired $t$-tests were used to assess between-session differences, alongside Bland-Altman analysis to assess systematic variations between the measurement values from the two sessions. All statistical tests were performed at a 0.05 level of significance. All statistical analysis was performed using the Statistical Package for the Social Sciences (SPSS) software (Version 25.0 for Macintosh).

\section{Results}

None of the participants dropped out of the study and all of them completed the three walking conditions over two sessions. Demographic information for all 25 able-bodied male participants is presented in Table 1.

Table 1. Demographic characteristics of the participants. BMI, body mass index.

\begin{tabular}{ccc}
\hline Demographic Measure & Mean \pm SD & Range \\
\hline Age (years) & $34.00 \pm 4.44$ & $30-46$ \\
Height $(\mathrm{m})$ & $1.71 \pm 0.06$ & $1.58-1.82$ \\
Weight $(\mathrm{kg})$ & $76.88 \pm 9.85$ & $57.60-100.2$ \\
BMI $\left(\mathrm{kg} / \mathrm{m}^{2}\right)$ & $26.17 \pm 2.48$ & $19.50-30.20$ \\
\hline
\end{tabular}

Descriptive results in Table 2 show that none of the symmetry scores for the medial-lateral, vertical, or anterior-posterior GRFs had perfect symmetry during free walking (no symmetry value was equal to one). This observation was consistent across walking conditions in both gait sessions. Furthermore, the paired $t$-tests revealed insignificant differences between the mean symmetry scores for the vertical, anterior-posterior, or medial-lateral GRFs recorded in both sessions across walking conditions ( $t$-test, $p>0.05)$. However, this was not the case for walking speed, which increased significantly under both dual-task walking conditions in session 2 ( $t$-test, $p=0.027$ during DT1 and 0.038 during DT2). Overall, the differences between the symmetry scores in both sessions were less than 0.02 , whilst the between-session differences of the speed parameters were between 0.032 and $0.059 \mathrm{~m} / \mathrm{s}$. The Bland-Altman plots (Figure 1) demonstrated that the between-session differences for all symmetry scores and speed parameters for the majority of the participants were located within the 95\% limits of agreement.

Multivariate ANOVA tests demonstrated that the interaction effect of sessions and walking conditions was not significant (Wilk's lambda $=0.881, \mathrm{~F}(8,17)=0.286, p=0.961$ ). The main effect of sessions was also insignificant (Wilk's lambda $=0.714, \mathrm{~F}(8,21)=2.102, p=0.117$ ), although the main effect of walking condition was significant (Wilk's lambda $=0.454, \mathrm{~F}(8,17)=2.555, p=0.050$ ). Of all outcome measurements, only the symmetry scores of the vertical GRF differed significantly across walking conditions $(p=0.003)$. Post hoc analyses revealed that significant differences occurred between FW and DT1 $(p=0.031)$, as well as between FW and DT2 $(p=0.019)$. Figure 2 presents the estimated marginal means of outcome measurements across walking conditions in both sessions. Figure 3 compares the means of the symmetry scores of all three GRFs across walking conditions in Session 2. 
Table 2. Descriptive statistics of the ground reaction force (GRF) symmetry and walking speed during free single-task walking (FW), and walking while concurrently performing the 1-back task (DT1) and the 2-back task (DT2) in two sessions.

\begin{tabular}{|c|c|c|c|c|c|c|c|c|}
\hline & & \multicolumn{2}{|c|}{ Session 1} & \multicolumn{2}{|c|}{ Session 2} & \multirow{2}{*}{$\begin{array}{c}\begin{array}{c}\text { Sessions } \\
\text { Combined }\end{array} \\
\text { Difference } \pm \text { SD }\end{array}$} & \multirow[t]{2}{*}{$t$-Test $p$} & \multirow[t]{2}{*}{$95 \% \mathrm{CI}$} \\
\hline & & Mean \pm SD & $\mathrm{CV} \%$ & Mean \pm SD & CV\% & & & \\
\hline \multirow{3}{*}{$\begin{array}{c}\text { Medial- } \\
\text { lateral }\end{array}$} & FW & $0.862 \pm 0.069$ & 8.0 & $0.880 \pm 0.059$ & 6.7 & $-0.018 \pm 0.063$ & 0.161 & -0.044 to 0.008 \\
\hline & DT1 & $0.861 \pm 0.068$ & 7.9 & $0.875 \pm 0.069$ & 7.9 & $-0.015 \pm 0.062$ & 0.252 & -0.040 to 0.011 \\
\hline & DT2 & $0.856 \pm 0.069$ & 8.1 & $0.863 \pm 0.070$ & 8.1 & $-0.008 \pm 0.066$ & 0.562 & -0.035 to 0.019 \\
\hline \multirow{3}{*}{ Vertical } & FW & $0.902 \pm 0.044$ & 4.9 & $0.893 \pm 0.044$ & 4.9 & $0.009 \pm 0.054$ & 0.421 & -0.014 to 0.031 \\
\hline & DT1 & $0.890 \pm 0.044$ & 4.9 & $0.879 \pm 0.038$ & 4.3 & $0.012 \pm 0.036$ & 0.116 & -0.003 to 0.027 \\
\hline & DT2 & $0.885 \pm 0.038$ & 4.3 & $0.878 \pm 0.037$ & 4.2 & $0.007 \pm 0.051$ & 0.490 & -0.014 to 0.028 \\
\hline \multirow{3}{*}{$\begin{array}{l}\text { Anterior- } \\
\text { posterior }\end{array}$} & FW & $0.847 \pm 0.090$ & 10.6 & $0.847 \pm 0.102$ & 12.0 & $-0.001 \pm 0.050$ & 0.950 & -0.021 to 0.020 \\
\hline & DT1 & $0.850 \pm 0.093$ & 10.9 & $0.854 \pm 0.112$ & 13.1 & $-0.003 \pm 0.062$ & 0.787 & -0.029 to 0.22 \\
\hline & DT2 & $0.839 \pm 0.101$ & 12.0 & $0.845 \pm 0.091$ & 10.8 & $-0.006 \pm 0.049$ & 0.553 & -0.026 to 0.014 \\
\hline \multirow{3}{*}{$\begin{array}{l}\text { Speed } \\
(\mathrm{m} / \mathrm{s})\end{array}$} & FW & $1.45 \pm 0.157$ & 9.0 & $1.48 \pm 0.170$ & 8.8 & $-0.032 \pm 0.117$ & 0.187 & -0.080 to 0.017 \\
\hline & DT1 & $1.40 \pm 0.139$ & 9.7 & $1.45 \pm 0.139$ & 8.8 & $-0.050 \pm 0.107$ & $0.027 *$ & -0.094 to -0.006 \\
\hline & DT2 & $1.39 \pm 0.182$ & 9.6 & $1.45 \pm 0.154$ & 9.0 & $-0.059 \pm 0.134$ & $0.038 *$ & -0.114 to -0.003 \\
\hline
\end{tabular}

Numbers in bold are statistically significant $\left({ }^{*} p<0.05\right)$. CV, coefficient of variation; CI, confidence interval.
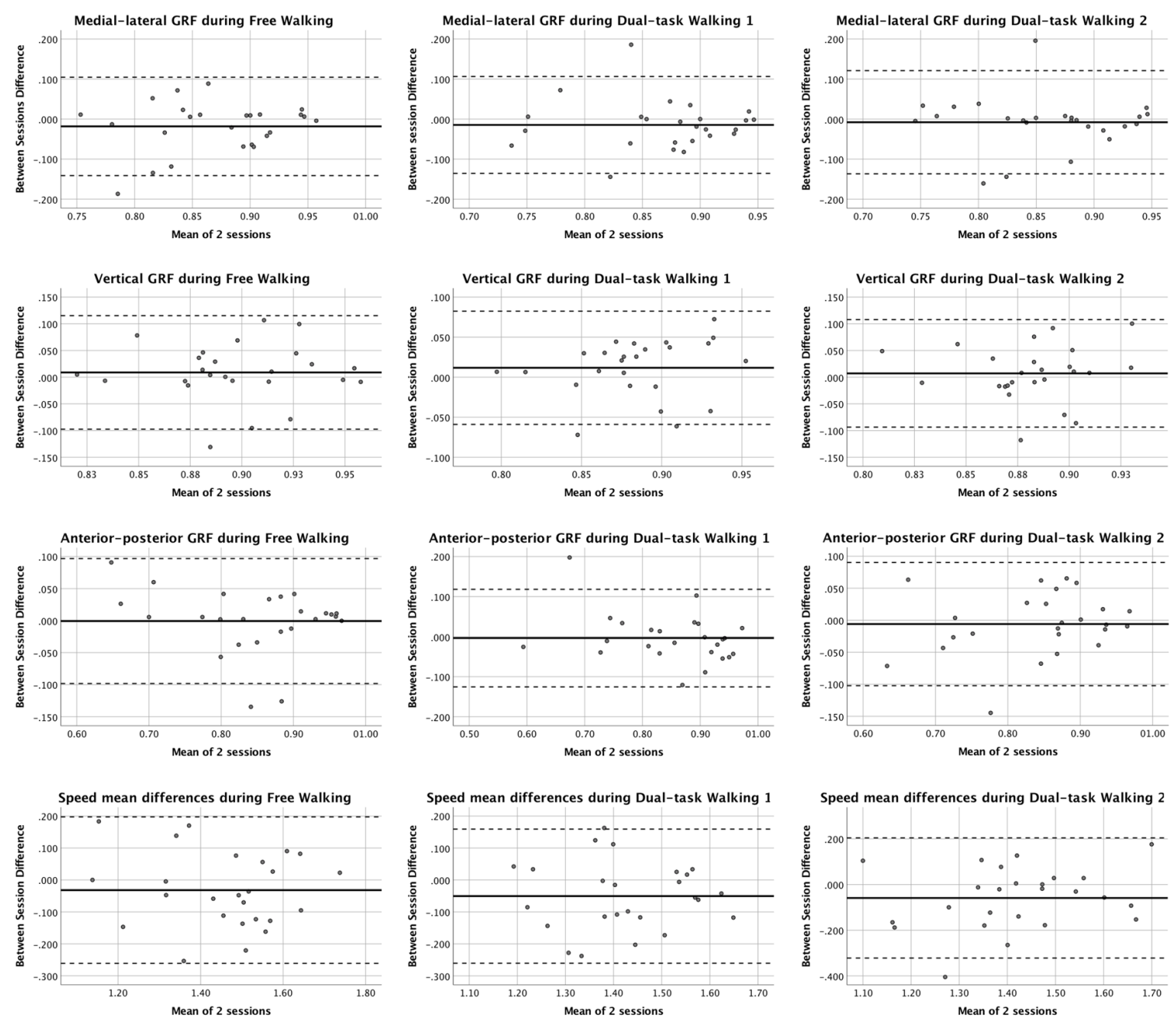

Figure 1. Bland-Altman plots for outcome measurements; the solid line represents the mean difference between the two sessions, while the upper and lower dashed lines represent the $95 \%$ limits of agreement. 

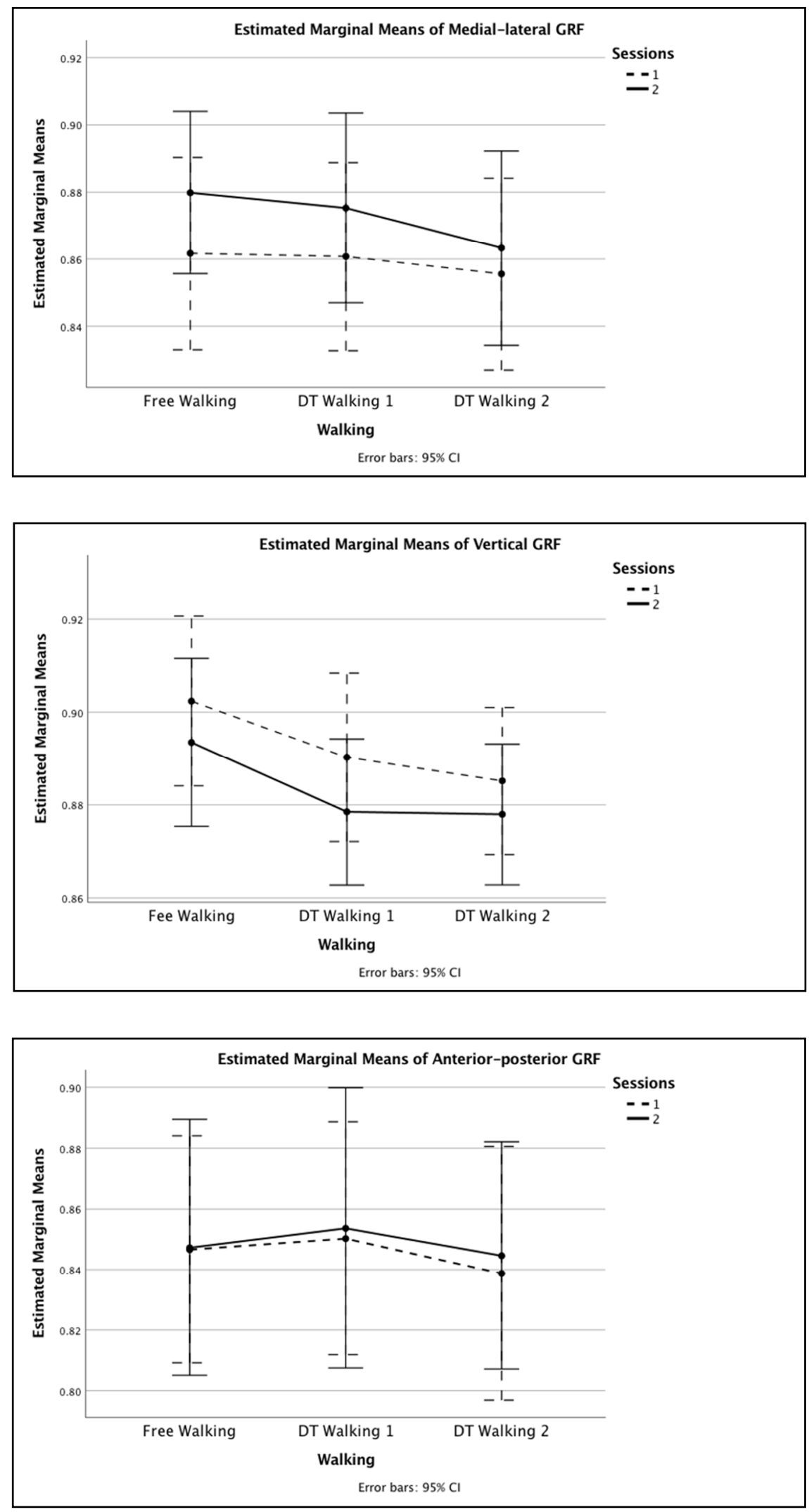

Figure 2. Cont. 


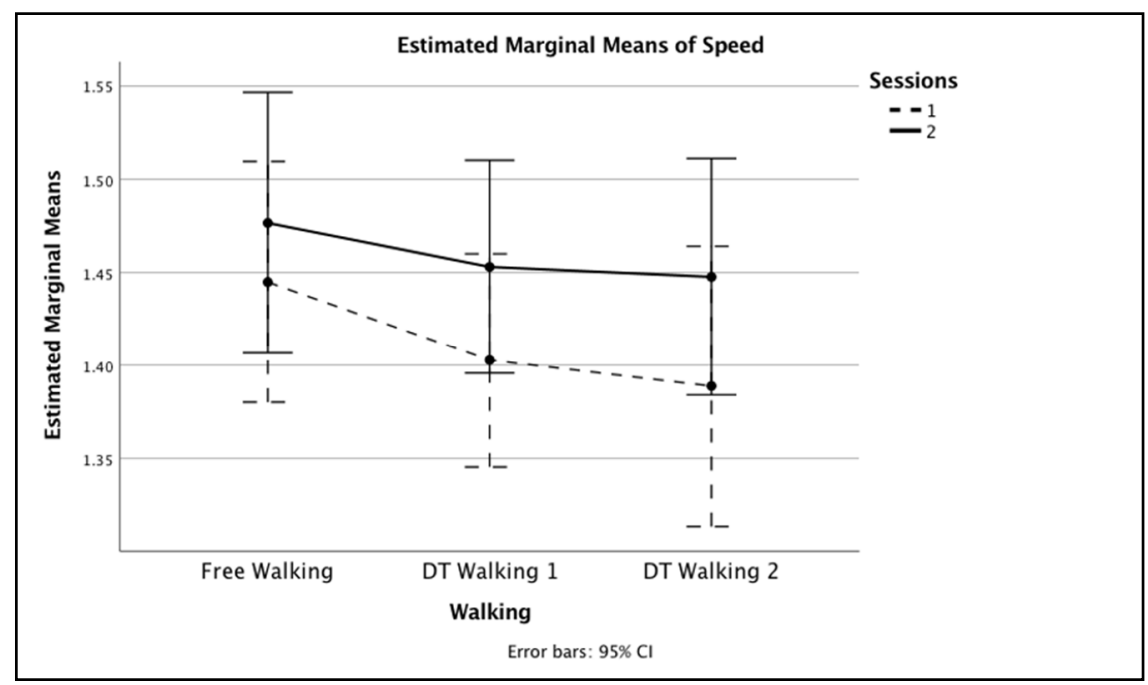

Figure 2. Estimated marginal means of outcome measurements across walking conditions in both sessions.

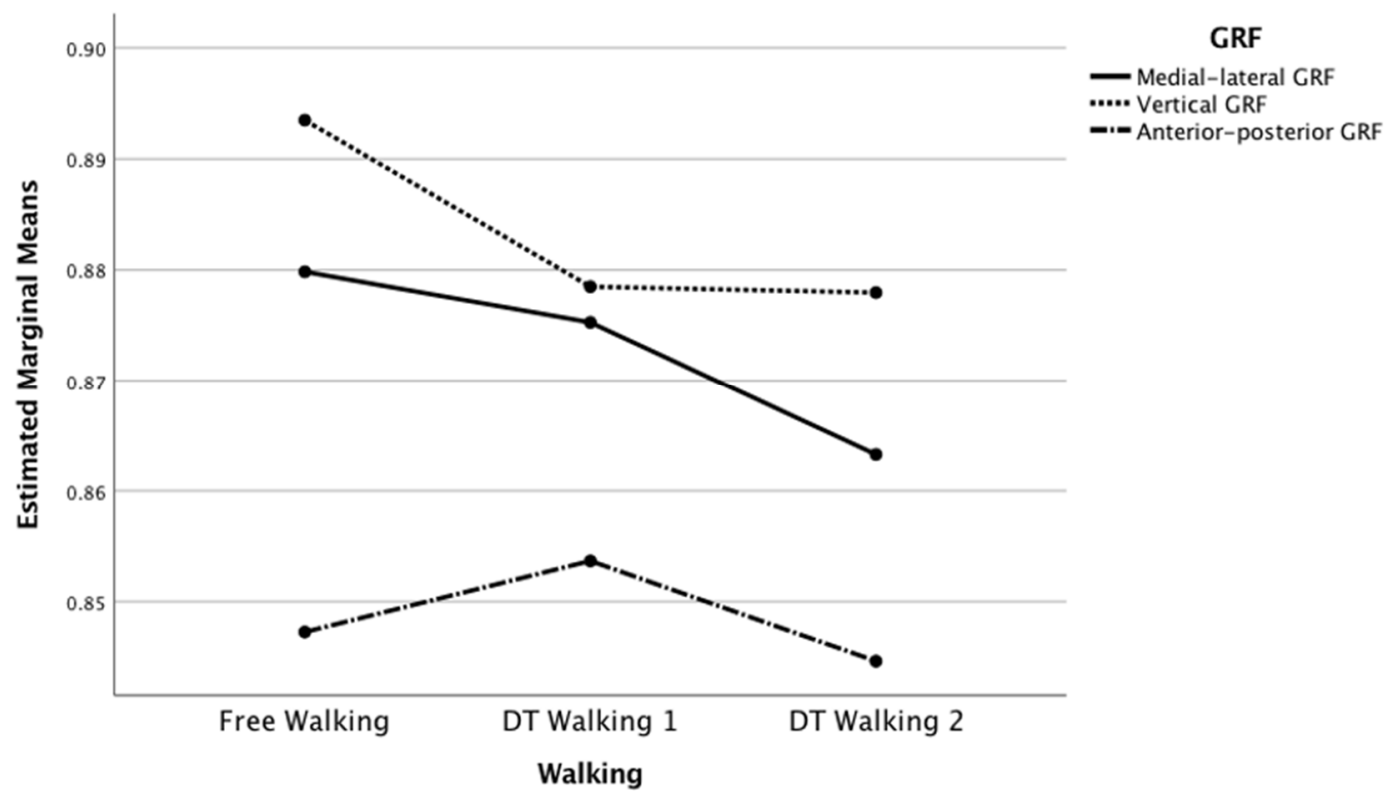

Figure 3. Mean plot of the symmetry scores of the three GRFs altogether across walking conditions in Session 2.

\section{Discussion}

This study sought to establish the symmetry characteristics of the three components of GRF and changes in walking speed in able-bodied individuals during walking with and without concurrent cognitive demands, over two gait assessment sessions. Our work suggests that there is imperfect symmetry in the medial-lateral, vertical, and anterior-posterior GRFs during any tested walking conditions. This supports the notion that certain levels of asymmetry within the able-bodied gait are physiologically acceptable [15]. For the symmetry between sessions, the results showed insignificant symmetry variations under all walking conditions. Inspection of the Bland-Altman graphs confirmed the agreement between the symmetry variables in the two sessions. These findings imply strong consistency of the symmetry variables in both sessions, providing evidence for the reliability of using the instrumented treadmill to obtain GRF parameters during self-paced walking in able-bodied individuals. 
A close examination of the symmetry scores and their variability proportions across walking conditions demonstrated that each GRF component has a unique symmetry behavior. Interestingly, the combination of symmetry and variability characteristics appears to define the function of each GRF component during gait. To illustrate, the vertical GRF exhibited the most symmetrical features and the least variability amongst all GRF components $(\mathrm{CV}<5 \%)$, which together promote gait steadiness in the able-bodied. The medial-lateral GRF displayed the second most symmetrical features of all GRF components, with a variability of less than $10 \%$ under all walking conditions. The symmetry of the anterior-posterior GRF presented the least symmetrical features and the highest variability compared to the other GRF components ( $\mathrm{CV}>10 \%$ ), which allow for adjustments in the acceleration/deceleration of the walking strides. Therefore, current findings demonstrated that imperfect symmetry is actually important to allow for gait variability and permit gait adaptability during different walking conditions. These findings are clinically relevant and can be associated with several important implications in clinical practice, including accurate diagnostic processes, monitoring patients' recovery toward normal gait, and other decision-making purposes (e.g., whether an orthotic or prosthetic device is suitable for supporting an individual's gait).

The vertical GRF in able-bodied individuals was thought to be perfectly symmetrical, with a deviation of less than 5\% during overground walking, whilst the medial-lateral GRF had the most substantial asymmetry $[7,9,12]$. Our findings suggested that the vertical GRF was symmetrical during free walking, but the anterior-posterior GRF depicted the least symmetrical characteristics of all GRF components. The discrepancy between our findings and those reported in the literature $[7,9,12]$ might be attributed to the methodology utilized. Previous studies commonly collected selected GRF parameters from a few nonconsecutive overground walking strides [7,9,12]. The study by Yiou et al. [41] indicated that the medial-lateral balance is challenged during walking initiation because of the decrease in the size of the base of support associated with stepping forward. This probably explains the high variability in the medial-lateral GRF in the previous studies [7,9]. The current study captured bilateral GRFs during self-paced consecutive walking strides and negated the effects of gait initiation and termination by processing pure continuous GRF waveforms. In view of that, our work provides more comprehensive findings than the existing literature.

To our knowledge, this study was also the first to investigate the effects of dual-task walking interference on the symmetry characteristics of the three GRF components. Our results demonstrated more asymmetry patterns during dual-tasking compared to a single-task walking condition. However, there was minimal interference with the symmetry of the anterior-posterior GRF under dual-task walking conditions. Paul et al. [42] indicated that the amount of deterioration in a given task under dual-task conditions demonstrates how much attention was needed to maintain the performance of that task and, hence, how automated it was. Thus, the changes within the symmetry of the anterior-posterior GRF under dual-task walking conditions provide insights into the dominance of automatic coordination processes in regulating the symmetry of this component. This, however, was not the case for the symmetry of the vertical GRF, in which the results revealed significant interference under concurrent challenges even though it was the component that had the most symmetrical features during free walking. The detrimental effects of dual-task walking on the symmetry of the vertical GRF increased when the concurrent cognitive task became more challenging with the 2-back memory task. These findings imply that the regulation of the symmetry of the vertical GRF is not primarily controlled by an automatic process, as it appears to be sensitive to the presence of additional cognitive loading. This could be due to the role of the vertical GRF during walking, which involves the acceleration of the body's mass over two alternative lower-limb movements while maintaining upright postural stability [43,44]. For the complexity of this function, central integration of multiple sensory input from the sensory and musculoskeletal systems is conceivably required to continuously modulate symmetry parameters with respect to the walking condition [44]. Our findings are in line with Yogev-Seligmann et al. [45], who suggested that bilateral coordination of limb movements makes gait especially vulnerable to cognitive loading during dual-tasking compared to standing and 
cycling. In our work, manipulating working memory with the $N$-back task during walking may also have contributed to sharing the neural correlates responsible for gait regulation [20,46], leading to a disproportionate change in symmetry. Further exploration is warranted to determine whether the significant increase in asymmetrical features of the vertical GRF during dual-task walking was an adaptive strategy to maintain gait stability or resulted from the competition of the concurrent processes for the attentional resources.

With regard to the effects of dual-task walking on speed parameters, previous studies frequently reported that dual-task walking significantly reduces gait speed in individuals with and without a pathological gait condition $[23,24,27,35]$. In line with these studies, the current study witnessed a reduction in the participants' average walking speed during both dual-task walking conditions; however, this reduction was not statistically significant. Interestingly, the current results also revealed an increment in the participants' walking speed during all walking conditions in session 2 compared to session 1 . This increment was statistically significant during both dual-task walking conditions but was not significant during the free walking condition. These findings suggest that the detrimental impact of dual-task walking on walking speed was significantly reduced in session 2 . Such findings are not surprising, given that the participants in our study were healthy individuals without any physical abnormality or cognitive impairments and were familiarized with the testing protocol in session 1 . The study by Nadkarni et al. [35], which introduced the 2-back task to comparable healthy young adults during dual-task walking, reported a significant reduction in their participants' walking speed, although their participants had practiced the cognitive tasks before walking trials. Nevertheless, the participants in Nadkarni et al.'s study [35] practiced the N-back task as a single task only in front of a computer screen in the same session during which gait measurements were recorded. The participants in the current study, in contrast, practiced the cognitive tasks while actively engaging in walking trials, and gait measurements were recorded during two gait assessment sessions on two different days. In our study, the practice of walking in isolation and with dual-task demands during session 1, in addition to the period between the two assessment sessions, may all have helped to form and consolidate a new procedural memory of the walking trials before taking measurements in session 2. The procedural memory plays a crucial role in supporting motor behavior and enhancing automaticity [47], and it appears to have mitigated the detrimental effects of dual-task performance on speed parameters. Future research is warranted to further investigate the role of procedural memory in reducing dual-task effects on gait performance.

The present study addressed several limitations in the existing literature concerning gait symmetry, which is often assessed and described in clinical practice to guide rehabilitation plans. Our work may be the first to provide insight into the symmetry of GRFs in able-bodied individuals under three walking conditions representing the challenges of daily walking. Despite the fact that the current findings can be generalized only to male able-bodied individuals, the study lacks confounding factors that may influence symmetry, such as age, gender, and walking speed. This study demonstrated the effects of cognitive loading on gait symmetry, although cautious interpretation of these results should be considered, as we only used the auditory $\mathrm{N}$-back test to manipulate cognitive loading during walking. Various cognitive tasks could impact gait symmetry differently. Further research is warranted to evaluate the effects of other cognitive tasks on gait symmetry in healthy individuals and patient populations.

\section{Conclusions}

In conclusion, this study yielded new evidence about the imperfect symmetry characteristics of the vertical, anterior-posterior, and medial-lateral GRFs in able-bodied gait. The results suggest that concurrent cognitive tasks during walking induced significant impacts on the vertical GRF. The between-session results reported in this study revealed the importance of practicing prior to dual-task assessments to reduce the detrimental effects of dual-task walking on gait. As self-paced gait treadmill use becomes more prevalent in gait analysis, the results of this study demonstrate 
the reliability of its GRFs and symmetry values, which can be used as criteria for comparing male individuals with and without gait pathology. Future work is still required to explore the potential effects of cognitive loading on gait symmetry in female individuals and in different age groups.

Author Contributions: Conceptualization, R.A.-J. and M.A.-A.; data curation, M.A.-A.; formal analysis, R.A.-J. and M.A.-A.; investigation, M.A.-A.; methodology, R.A.-J. and M.A.-A.; project administration, R.A.-J and M.A.-A.; resources, M.A.-A.; software, M.A.-A.; supervision, M.A.-A.; validation, M.A.-A.; visualization, R.A.-J.; writing-original draft, R.A.-J.; writing_review and editing, R.A.-J. and M.A.-A. All authors read and approved the final manuscript.

Funding: This research received no external funding. The APC was funded by Cardiff University Institutional Open Access Fund.

Acknowledgments: The authors would like to thank all volunteers for their participation in this study. The authors would like to thank Abdulrhman Mashabi and Hilal Al Balushi for their help in the data collection and Mark Postans for his guidance on the use of the $N$-back task. The authors would also like to thank their colleagues who provided assistance and contribution to the MATLAB code regarding symmetry, as well as for their constructive feedback on the manuscript.

Conflicts of Interest: The authors declare no conflict of interest.

\section{References}

1. Nasirzade, A.; Sadeghi, H.; Mokhtarinia, H.R.; Rahimi, A. A review of selected factors affecting gait symmetry. Phys. Treat. Specif. Phys. Ther. 2017, 7, 3-12. [CrossRef]

2. Hsiao-Wecksler, E.T.; Polk, J.D.; Rosengren, K.S.; Sosnoff, J.J.; Hong, S. A review of new analytic techniques for quantifying symmetry in locomotion. Symmetry 2010, 2, 1135-1155. [CrossRef]

3. Ankarali, M.M.; Sefati, S.; Madhav, M.; Long, A.; Bastian, A.; Cowan, N. Walking dynamics are symmetric (enough). J. R. Soc. Interface 2015, 12, 20150209. [CrossRef] [PubMed]

4. Wu, J.; Wu, B. The novel quantitative technique for assessment of gait symmetry using advanced statistical learning algorithm. Biomed Res. Int. 2015, 2015, 528971. [CrossRef] [PubMed]

5. Toro, B.; Nester, C.; Farren, P. A review of observational gait assessment in clinical practice. Physiother. Theory Pract. 2009, 19, 137-149. [CrossRef]

6. Baker, R.; Esquenazi, A.; Benedetti, M.G.; Desloovere, K. Gait analysis: Clinical facts. Eur. J. Phys. Rehabil. Med. 2016, 52, 560-574.

7. Herzog, W.; Nigg, B.M.; Read, L.J.; Olsson, E. Asymmetries in ground reaction force patterns in normal human gait. Med. Sci. Sports Exerc. 1988, 21, 1689-1699. [CrossRef]

8. Seeley, M.K.; Umberger, B.R.; Shapiro, R. A test of the functional asymmetry hypothesis in walking. Gait Posture 2008, 28, 24-28. [CrossRef]

9. Giakas, G.; Baltzopoulos, V. Time and frequency domain analysis of ground reaction forces during walking: An investigation of variability and symmetry. Gait Posture 1997, 5, 189-197. [CrossRef]

10. Forczek, W.; Staszkiewicz, R. An evaluation of symmetry in the lower limb joints during the able-bodied gait of women and men. J. Hum. Kinet. 2012, 35, 47-57. [CrossRef]

11. Sadeghi, H. Local or global asymmetry in gait of people without impairments. Gait Posture 2003, 17, $197-204$. [CrossRef]

12. Burnett, D.R.; Campbell-Kyureghyan, N.H.; Cerrito, P.B.; Quesada, P.M. Symmetry of ground reaction forces and muscle activity in asymptomatic subjects during walking, sit-to-stand, and stand-to-sit tasks. J. Electromyogr. Kinesiol. 2011, 21, 610-615. [CrossRef] [PubMed]

13. Viteckova, S.; Kutilek, P.; Svoboda, Z.; Krupika, R.; Kauler, J.; Szabo, Z. Gait symmetry measures: A review of current and prospective methods. Biomed. Signal Process. Control 2018, 42, 89-100. [CrossRef]

14. Jeleń, P.; Wit, A.; Dudziński, K.; Nolan, L. Expressing gait-line symmetry in able-bodied gait. Dyn. Med. 2008, 7, 1-9. [CrossRef] [PubMed]

15. Vaverka, F.; Elfmark, M.; Svoboda, Z.; Janura, M. System of gait analysis based on ground reaction force assessment. Acta Gymnica 2015, 45, 187-193. [CrossRef]

16. Wang, Y.; Watanabe, K. Symmetry and variability of vertical ground reaction force and center of pressure in able-bodied gait. J. Appl. Biomech. 2012, 28, 473-478. [CrossRef] 
17. Plotnik, M.; Bartsch, R.P.; Zeev, A.; Giladi, N.; Hausdorff, J.M. Effects of walking speed on asymmetry and bilateral coordination of gait. Gait Posture 2013, 38, 864-869. [CrossRef]

18. Al-Amri, M.; Al Balushi, H.; Mashabi, A. Intra-rater repeatability of gait parameters in healthy adults during self-paced treadmill-based virtual reality walking. Comput. Methods Biomech. Biomed. Engin. 2017, 20, 1669-1677. [CrossRef]

19. Luigi Tesio, V.R. Gait analysis on split-belt force treadmills: Validation of an instrument. Am. J. Phys. Med. Rehabil. 2008, 87, 515-526. [CrossRef]

20. Yogev-Seligmann, G.; Hausdorff, J.M.; Giladi, N. The role of executive function and attention in gait. Mov. Disord. 2008, 23, 329-342. [CrossRef]

21. Mendel, T.; Barbosa, W.O.; Sasaki, A.C. Dual task training as a therapeutic strategy in neurologic physical therapy: A literature review. Acta Fisiátrica 2015, 22, 206. [CrossRef]

22. Gillain, S.; Boutaayamou, M.; Schwartz, C.; Dardenne, N.; Bruyère, O.; Brüls, O.; Croisier, J.-L.; Salmon, E.; Reginster, J.-Y.; Garraux, G.; et al. Gait symmetry in the dual task condition as a predictor of future falls among independent older adults: A 2-year longitudinal study. Aging Clin. Exp. Res. 2019, 3, 1057-1067. [CrossRef] [PubMed]

23. Al-Yahya, E.; Dawes, H.; Smith, L.; Dennis, A.; Howells, K.; Cockburn, J. Cognitive motor interference while walking: A systematic review and meta-analysis. Neurosci. Biobehav. Rev. 2011, 35, 715-728. [CrossRef] [PubMed]

24. Hollman, J.H.; Kovash, F.M.; Kubik, J.J.; Linbo, R.A. Age-related differences in spatiotemporal markers of gait stability during dual task walking. Gait Posture 2007, 26, 113-119. [CrossRef]

25. Schaefer, S.; Lindenberger, U. Thinking while walking: Experienced high-heel walkers flexibly adjust their gait. Front. Psychol. 2013, 4, 1-7. [CrossRef]

26. Yogev, G.; Plotnik, M.; Peretz, C.; Giladi, N.; Hausdorff, J.M. Gait asymmetry in patients with Parkinson's disease and elderly fallers: When does the bilateral coordination of gait require attention? Exp. Brain Res. 2007, 177, 336-346. [CrossRef]

27. Plotnik, M.; Giladi, N.; Hausdorff, J.M. Bilateral coordination of gait and Parkinson's disease: The effects of dual tasking. J. Neurol. Neurosurg. Psychiatry 2009, 80, 347-350. [CrossRef]

28. Ribeiro, T.S.; de Sousa, A.C.; de Lucena, L.C.; Santiago, L.M.; Lindquist, A.R. Does dual task walking affect gait symmetry in individuals with Parkinson's disease? Eur. J. Physiother. 2018, 21, 8-14. [CrossRef]

29. Faul, F.; Erdfelder, E.; Axel, B.; Lang, A.-G. Statistical power analyses using G*Power 3.1: Tests for correlation and regression analyses. Behav. Res. Methods 2009, 41, 1149-1160. [CrossRef]

30. Kimura, N.; van Deursen, R. The effect of visual dual-tasking interference on walking in healthy young adults. Gait Posture 2020, 79, 80-85. [CrossRef]

31. Van den Bogert, A.J.; Geijtenbeek, T.; Even-Zohar, O.; Steenbrink, F.; Hardin, E.C. A real-time system for biomechanical analysis of human movement and muscle function. Med. Biol. Eng. Comput. 2013, 51, 1069-1077. [CrossRef] [PubMed]

32. Sloot, L.H.; van der Krogt, M.M.; Harlaar, J. Effects of adding a virtual reality environment to different modes of treadmill walking. Gait Posture 2014, 39, 939-945. [CrossRef] [PubMed]

33. Meyer, C.; Kileen, T.; Easthope, C.; Curt, A.; Bollinger, M.; Linnebank, M.; Zörner, B.; Filley, L. Familiarization with treadmill walking: How much is enough? Sci. Rep. 2019, 9, 1-10. [CrossRef] [PubMed]

34. Seltman, H. Experimental Design and Analysis. 2018. Available online: http://www.stat.cmu.edu/ \{\}hseltman/ 309/Book/Book.pdf (accessed on 28 November 2020).

35. Nadkarni, N.K.; Zabjek, K.; Lee, B.; McIlroy, W.E.; Black, S.E. Effect of working memory and spatial attention tasks on gait in healthy young and older adults. Mot. Control 2010, 14, 195-210. [CrossRef]

36. Monk, A.F.; Jackson, D.; Nielsen, D.; Jefferies, E.; Olivier, P. N-backer: An auditory n-back task with automatic scoring of spoken responses. Behav. Res. Methods 2011, 43, 888-896. [CrossRef]

37. Lövdén, M.; Schaefer, S.; Pohlmeyer, A.E.; Lindenberger, U. Walking variability and working-memory load in aging: A dual-process account relating cognitive control to motor control performance. J. Gerontol. B Psychol. Sci. Soc. Sci. 2008, 63, 121-128. [CrossRef]

38. Liao, F.; Wang, J.; He, P. Multi-resolution entropy analysis of gait symmetry in neurological degenerative diseases and amyotrophic lateral sclerosis. Med. Eng. Phys. 2008, 30, 299-310. [CrossRef]

39. Robinson, R.O.; Herzog, W.; Nigg, B.M. Use of force platform variables to quantify the effects of chiropractic manipulation on gait symmetry. J. Manip. Physiol. Ther. 1987, 10, 172-176. 
40. Liikavainio, T.; Isolehto, J.; Helminen, H.J.; Perttunen, J.; Lepola, V.; Kiviranta, I.; Arokoski, J.P.A.; Komi, P.V. Loading and gait symmetry during level and stair walking in asymptomatic subjects with knee osteoarthritis: Importance of quadriceps femoris in reducing impact force during heel strike? Knee 2007, 14, 231-238. [CrossRef]

41. Yiou, E.; Caderby, T.; Delafontaine, A.; Fourcade, P.; Honeine, J.L. Balance control during gait initiation: State-of-the-art and research perspectives. World J. Orthop. 2017, 8, 815-828. [CrossRef]

42. Paul, S.S.; Ada, L.; Canning, C.G. Automaticity of walking-Implications for physiotherapy practice. Phys. Ther. Rev. 2005, 10, 15-23. [CrossRef]

43. Marasović, T.; Cecić, M.; Zanchi, V. Analysis and interpretation of ground reaction forces in normal gait. WSEAS Trans. Syst. 2009, 8, 1105-1114.

44. Dos Santos, D.A.; Fukuchi, C.A.; Fukuchi, R.K.; Duarte, M. A data set with kinematic and ground reaction forces of human balance. PeerJ 2017, 5, e3626. [CrossRef] [PubMed]

45. Yogev-Seligmann, G.; Giladi, N.; Gruendlinger, L.; Hausdorff, J.M. The contribution of postural control and bilateral coordination to the impact of dual tasking on gait. Exp. Brain Res. 2013, 226, 81-93. [CrossRef] [PubMed]

46. Owen, A.M.; McMillan, K.M.; Laird, A.R.; Bullmore, E. N-Back working memory paradigm: A meta-analysis of normative functional neuroimaging studies. Hum. Brain Mapp. 2005, 25, 46-59. [CrossRef] [PubMed]

47. Studer, M.; Winningham, R. Retraining automaticity: Recovering the procedural memory of walking after stroke. Phys. Med. Rehabil. Int. 2017, 4, 1125. [CrossRef]

Publisher's Note: MDPI stays neutral with regard to jurisdictional claims in published maps and institutional affiliations.

(C) 2020 by the authors. Licensee MDPI, Basel, Switzerland. This article is an open access article distributed under the terms and conditions of the Creative Commons Attribution (CC BY) license (http://creativecommons.org/licenses/by/4.0/). 